\title{
Common fixed point results via simulation type functions in non-Archimedean modular metric spaces and applications
}

https://doi.org/10.1515/taa-2020-0109

Received 29 September, 2021; accepted 7 January, 2022

\begin{abstract}
In this study, we demonstrate the existence and uniqueness of common fixed points of a generalized $(\alpha, \beta)$ - simulation contraction on a non-Archimedean modular metric space. We achieve some consequences in non-Archimedean modular metric spaces as an application, using the structure of a directed graph. Eventually, we contemplate the existence of solutions to a class of functional equations standing up dynamic programming with the help of our outcomes.
\end{abstract}

Keywords: Non-Archimedean modular metric space, Simulation function, Cyclic $(\alpha, \beta)$-admissible pair

MSC: 47H10, 54H25, 37C25

\section{Introduction}

Subsequently, the letters $\mathbb{R}, \mathbb{R}_{+}$, and $\mathbb{N}$ will specify the set of all real numbers, the set of all nonnegative real numbers, and the set of all positive integer numbers, respectively.

In 2010, Chistyakov [1, 2] set up a new structure named modular metric, which is an extension of metric and a linear modular.

Definition 1.1 $[1,3]$ Let $M$ be non-void set and $\delta:(0, \infty) \times M \times M \rightarrow[0, \infty]$ be a function fulfilling the following statements: for all $\lambda, \mu>0$ and $p, q, k \in M$,

$\delta_{1} . p=q$ if and only if $\delta_{\lambda}(p, q)=0$;

$\delta_{2} . \delta_{\lambda}(p, q)=\delta_{\lambda}(p, q)$;

$\delta_{3} . \delta_{\lambda+\mu}(p, q) \leq \delta_{\lambda}(p, k)+\delta_{\mu}(k, q)$.

So, $\delta$ is entitled modular metric in $M$, and in the present case, $M_{\delta}$ is a modular metric space. If we substitute $\left(\delta_{1}\right)$ by

$\delta_{4} \cdot \delta_{\lambda}(p, p)=0$ for all $\lambda>0$ and $p \in M$,

then $\delta$ is said to be a pseudomodular metric on $M$. A modular metric $\delta$ on $M$ is named regular if the following weaker version of $\left(\delta_{1}\right)$ is provided for some $\lambda>0$;

$\delta_{5} . p=q$ if and only if $\delta_{\lambda}(p, q)=0$.

As well, $\delta$ is a convex function if, for $\lambda, \mu>0$ and $p, q, k \in M$, the inequality holds:

$\delta_{6} . \delta_{\lambda+\mu}(p, q) \leq \frac{\lambda}{\lambda+\mu} \delta_{\lambda}(p, k)+\frac{\mu}{\lambda+\mu} \delta_{\mu}(k, q)$.

For all $\lambda, \mu>0$ and $p, q, k \in M_{\delta}$, if the following property

$\delta_{7} . \delta_{\max \{\lambda, \mu\}}(p, q) \leq \delta_{\lambda}(p, k)+\delta_{\mu}(k, q)$

`Corresponding Author: Mahpeyker Öztürk: Department of Mathematics, Sakarya University, Sakarya, Turkey, E-mail: mahpeykero@sakarya.edu.tr

Ekber Girgin: Department of Mathematics, Sakarya University, Sakarya, Turkey, E-mail: girginekber@gmail.com 
is put in a place of $\left(\delta_{3}\right)$, then, we attain a new space with some new features. This new structure $M_{\delta}$ is named a non-Archimedean modular metric space. For simplicity, throughout the study, we will write:

$$
\delta_{\lambda}(p, q)=\delta(\lambda, p, q)
$$

for all $\lambda>0$ and $p, q \in M$.

Definition 1.2 [1, 2] Presume that the function $\delta$ be a pseudomodular on $M$ and $\delta_{0} \in M$ be a fixed element. The hereinbelow two sets $M_{\delta}$ and $M_{\delta}^{\star}$ are called modular spaces (around $\delta_{0}$ ).

$$
M_{\delta}=M_{\delta}\left(p_{0}\right)=\left\{p \in M: \delta_{\lambda}\left(p, p_{0}\right) \text { as } \lambda \rightarrow \infty\right\}
$$

and

$$
M_{\delta}^{\star}=M_{\delta}^{\star}\left(p_{0}\right)=\left\{p \in M: \exists \lambda=\lambda(p)>0 \quad \text { such that } \delta_{\lambda}\left(p, p_{0}\right)<\infty\right\} .
$$

It is noticeable that $M_{\delta} \subset M_{\delta}^{\star}$, but this inclusion may be proper in general. Presume that from [1, 2], $\delta$ is modular on $M$; we derive that the modular space $M_{\delta}$ can be equipped with a (nontrivial) metric, induced by $\delta$ and presented by:

$$
d_{\delta}(p, q)=\inf \left\{\lambda>0: \delta_{\lambda}(p, q)<\lambda\right\}
$$

for all $p, q \in M_{\delta}$.

Note that if $\delta$ is a convex modular on $M$, then according to [1, 2], the two modular spaces coincide, i.e., $M_{\delta}=M_{\delta}^{\star}$, and this common set can be endowed with the metric $d_{\delta}^{\star}$ given by:

$$
d_{\delta}^{\star}(p, q)=\inf \left\{\lambda>0: \delta_{\lambda}(p, q)<1, \forall p, q \in M_{\delta}\right\},
$$

which is called Luxemburg distance.

Definition 1.3 [4] Let $M_{\delta}$ be a modular metric space, $S$ be a subset of $M_{\delta}$ and $\left(p_{n}\right)_{n \in \mathbb{N}}$ be a sequence in $M_{\delta}$. Then,

i. A sequence $\left(p_{n}\right)_{n \in \mathbb{N}}$ is called $\delta$-convergent to $p \in M_{\delta}$ if and only if $\delta_{\lambda}\left(p_{n}, p\right) \rightarrow 0$ as $n \rightarrow \infty$ for all $\lambda>0$, $p$ is said to be the $\delta$-limit of $\left(p_{n}\right)$.

ii. A sequence $\left(p_{n}\right)_{n \in \mathbb{N}}$ is called $\delta$-Cauchy if $\delta_{\lambda}\left(p_{n}, p_{m}\right) \rightarrow 0$, as $m, n \rightarrow \infty$ for all $\lambda>0$.

iii. A subset $S$ is called $\delta$-complete if any $\delta$-Cauchy sequence in $S$ is $\delta$-convergent to the point of $S$.

Definition 1.4 [4] Let $M_{\delta}$ be a modular metric space. The mapping $K: M_{\delta} \rightarrow M_{\delta}$ is $\delta$-continuous if $\delta_{\lambda}\left(p_{n}, p\right) \rightarrow 0$, provided to $\delta_{\lambda}\left(K p_{n}, K p\right) \rightarrow 0$ as $n \rightarrow \infty$.

Definition 1.5 [5] A simulation function is a mapping $\zeta:[0, \infty)^{2} \rightarrow \mathbb{R}$ admitting the following features:

$\zeta_{1} \cdot \zeta(0,0)=0$,

$\zeta_{2} . \zeta(p, q)<p-q$, for all $p, q>0$,

$\zeta_{3}$. if $\left\{q_{n}\right\}$ and $\left\{p_{n}\right\}$ are sequences in $(0, \infty)$ such that $\lim _{n \rightarrow \infty} q_{n}=\lim _{n \rightarrow \infty} p_{n}=l$ and $l \in(0, \infty)$, then

$$
\lim \sup _{n \rightarrow \infty} \zeta\left(q_{n}, p_{n}\right)<0
$$

Argoubi et al.[6] modified the definition of simulation function and so introduced it as:

Definition 1.6 A simulation function is a mapping $\zeta:[0, \infty)^{2} \rightarrow \mathbb{R}$ satisfying the followings:

i. $\zeta(p, q)<p-q$, for all $p, q>0$,

ii. if $\left\{q_{n}\right\}$ and $\left\{p_{n}\right\}$ are sequences in $(0, \infty)$ such that $\lim _{n \rightarrow \infty} q_{n}=\lim _{n \rightarrow \infty} p_{n}>0$, and $q_{n}<p_{n}$, then $\lim \sup \zeta\left(q_{n}, p_{n}\right)<$ 0.

Example 1.7 [6] The function $\zeta_{c}:[0, \infty) \times[0, \infty) \rightarrow \mathbb{R}$ is a simulation function specified by

$$
\zeta_{c}(q, p)= \begin{cases}1 & \text { if }(q, p)=(0,0) \\ c p-q & \text { otherwise }\end{cases}
$$

where $c \in(0,1)$.

One may point to [5]-[12] for some detailed information about simulation functions.

Definition 1.8 [13] Let $G:[0, \infty)^{2} \rightarrow \mathbb{R}$ is called $C$-class function when it has continuity property and admits the following features: 
i. $\quad G(p, q) \leq p$,

ii. $G(p, q)=p$ implies that either $p=0$ or $q=0$, for all $p, q \in[0, \infty)$.

Definition 1.9 [14] Let $\zeta:[0, \infty)^{2} \rightarrow \mathbb{R}$ be a function admitting the following features:

a. $\zeta(q, p)<G(p, q)$ for all $t, s>0$, where $G:[0, \infty)^{2} \rightarrow \mathbb{R}$ is a $C$-class function,

$b$. if $\left\{q_{n}\right\}$ and $\left\{p_{n}\right\}$ are sequences in $(0, \infty)$ such that $\lim _{n \rightarrow \infty} q_{n}=\lim _{n \rightarrow \infty} p_{n}>0$, and $q_{n}<p_{n}$, then $\lim \sup \zeta\left(q_{n}, p_{n}\right)<$ $C_{G}$.

Then $\zeta$ is named a $C_{G}$-simulation function.

Definition 1.10 [14] A mapping $G:[0, \infty)^{2} \rightarrow \mathbb{R}$ has the property $C_{G}$, if there exists a $C_{G} \geq 0$ such that

i. $G(p, q)>C_{G}$ implies $p>q$,

ii. $G(q, q) \leq C_{G}$ for all $q \in[0, \infty)$.

Let $T_{G}$ denotes the family of all $C_{G}$-simulation functions $\zeta:[0, \infty)^{2} \rightarrow \mathbb{R}$.

Recently, Alizadeh et al. [15] defined the concept of cyclic $(\alpha, \beta)$-admissible mapping as indicated below:

Definition 1.11 Let $M$ be a non-void set, $K$ be a self-mapping on $M$ and $\alpha, \beta: M \rightarrow[0, \infty)$ be two mappings.

$K$ is named a cyclic $(\alpha, \beta)$-admissible mapping if

(i) for some $p \in M, \alpha(p) \geq 1$ implies $\beta(K p) \geq 1$;

(ii) for some $p \in M, \beta(p) \geq 1$ implies $\alpha(K p) \geq 1$.

Besides, they demonstrated fixed point theorems by using the structure of cyclic $(\alpha, \beta)$-admissible mapping. Example 1.12 [15] Let $K: \mathbb{R} \rightarrow \mathbb{R}$ be a function described as $K p=-\left(p+p^{3}\right)$. Presume that $\alpha, \beta: \mathbb{R} \rightarrow \mathbb{R}_{+}$are furnishing mappings for all $p, q \in \mathbb{R}_{+}$such that

$$
\alpha(p)=e^{p} \text { and } \beta(q)=e^{-q} .
$$

Then $K$ is a cyclic $(\alpha, \beta)$-admissible mapping.

The generalized cyclic $(\alpha, \beta)$-admissible mappings have been generalized by Latif et al. [16], as follows.

Definition 1.13 Let $M$ be a non-void set, $K, L$ be two self-mappings on $M$ and $\alpha, \beta: M \rightarrow[0, \infty)$ be two mappings. $(K, L)$ is called a cyclic $(\alpha, \beta)$-admissible pair if

(i) for some $p \in M \alpha(p) \geq 1$ implies $\beta(K p) \geq 1$;

(ii) for some $p \in M \beta(p) \geq 1$ implies $\alpha(L p) \geq 1$.

Remark 1.16 In the above definition, if we acquire $K=L$, then $K$ is a cyclic $(\alpha, \beta)$-admissible mapping. Throughout this study, $\delta$ will be used as a convex and regular function.

\section{Main results}

Initially, this section aims to innovate the generalized $(\alpha, \beta)$-simulation contraction. Besides, common fixed point results are procured satisfying generalized $(\alpha, \beta)$-simulation contraction in non-Archimedean modular metric spaces.

Definition 2.1 Let $M_{\delta}$ be a non-Archimedean modular metric space, $K, L: M_{\delta} \rightarrow M_{\delta}$ be two self-mappings and $\alpha, \beta: M_{\delta} \rightarrow[0, \infty)$ be two functions. The pair $(K, L)$ is named a generalized $(\alpha, \beta)$-simulation contraction if there exists $\zeta \in T_{G}$ such that

$$
\alpha(p) \beta(q) \geq 1 \quad \Rightarrow \quad \zeta\left(\delta_{\lambda}(K p, L q), S(p, q)\right) \geq C_{G},
$$

where $S(p, q)=\max \left\{\delta_{\lambda}(p, q), \delta_{\lambda}(p, K p), \delta_{\lambda}(q, L q), \frac{\delta_{\lambda}(p, L q)+\delta_{\lambda}(q, K p)}{2}\right\}$, for all $p, q \in M_{\delta}$.

Theorem 2.2 Let $M_{\delta}$ be a $\delta$-complete non-Archimedean modular metric space and $K$ and $L$ are generalized $(\alpha, \beta)$-simulation contraction. Presume that the following circumstances hold:

i. $(K, L)$ is a cyclic $(\alpha, \beta)$-admissible pair,

ii. there exists $p_{0} \in M_{\delta}$ such that $\alpha\left(p_{0}\right) \geq 1$,

iii. $K$ or $L$ is a $\delta$-continuous mapping,

iv. if $\left\{p_{n}\right\}$ is a sequence in $M_{\delta}$ such that $p_{n} \rightarrow p$ and $\alpha\left(p_{2 n}\right) \geq 1, \beta\left(p_{2 n-1}\right) \geq 1$ for all $n \in \mathbb{N}$, then $\alpha(p) \geq 1$ and $\beta(p) \geq 1$. 
Then $K$ and $L$ hold a common fixed point. Additionally, if $\alpha(p) \beta(q) \geq 1$ for all $p, q \in$ Fix $(K, L)$, then $K$ and $L$ hold a unique common fixed point.

Proof Let $p_{0} \in M_{\delta}$ be such that $\alpha\left(p_{0}\right) \geq 1$. We will construct a sequence $\left\{p_{n}\right\}$ in $M_{\delta}$ by

$$
\begin{aligned}
& p_{2 n+2}=K p_{2 n+1}, \\
& p_{2 n+1}=L p_{2 n},
\end{aligned}
$$

for all $n \in \mathbb{N}$. Also, as $(K, L)$ is a cyclic $(\alpha, \beta)$-admissible pair and $\alpha\left(p_{0}\right) \geq 1$, then

$$
\beta\left(p_{1}\right)=\beta\left(K p_{0}\right) \geq 1
$$

which implies

$$
\alpha\left(p_{2}\right)=\alpha\left(L p_{1}\right) \geq 1 .
$$

By proceeding with this process, we get $\alpha\left(p_{2 n}\right) \geq 1$ and $\beta\left(p_{2 n+1}\right) \geq 1$ for all $n \in \mathbb{N}$. Thus, $\alpha\left(p_{2 n}\right) \beta\left(p_{2 n+1}\right) \geq 1$ for all $n \in \mathbb{N}$. From (1), we have

$$
\begin{aligned}
C_{G} & \leq \zeta\left(\delta_{\lambda}\left(K p_{2 n}, L p_{2 n+1}\right), S\left(p_{2 n}, p_{2 n+1}\right)\right) \\
& =\zeta\left(\delta_{\lambda}\left(p_{2 n+1}, p_{2 n+2}\right), S\left(p_{2 n}, p_{2 n+1}\right)\right) \\
& <G\left(S\left(p_{2 n}, p_{2 n+1}\right), \delta_{\lambda}\left(p_{2 n+1}, p_{2 n+2}\right)\right) .
\end{aligned}
$$

Further, using (i) of Definition 1.10, we achieve

$$
\delta_{\lambda}\left(p_{2 n+1}, p_{2 n+2}\right)<S\left(p_{2 n}, p_{2 n+1}\right),
$$

where

$$
\begin{aligned}
S\left(p_{2 n}, p_{2 n+1}\right)= & \max \left\{\delta_{\lambda}\left(p_{2 n}, p_{2 n+1}\right), \delta_{\lambda}\left(p_{2 n}, K p_{2 n}\right), \delta_{\lambda}\left(p_{2 n+1}, L p_{2 n+1}\right),\right. \\
& \left.\frac{\left(p_{2 n}, L p_{2 n+1}\right)+\left(p_{2 n+1}, K p_{2 n}\right)}{2}\right\} \\
& =\max \left\{\delta_{\lambda}\left(p_{2 n}, p_{2 n+1}\right), \delta_{\lambda}\left(p_{2 n}, p_{2 n+1}\right), \delta_{\lambda}\left(p_{2 n+1}, p_{2 n+2}\right),\right. \\
& \left.\frac{\delta_{\lambda}\left(p_{2 n}, p_{2 n+2}\right)+\delta_{\lambda}\left(p_{2 n+1}, p_{2 n+1}\right)}{2}\right\} \\
& =\max \left\{\delta_{\lambda}\left(p_{2 n}, p_{2 n+1}\right), \delta_{\lambda}\left(p_{2 n}, p_{2 n+1}\right), \delta_{\lambda}\left(p_{2 n+1}, p_{2 n+2}\right),\right. \\
& \left.\frac{\delta_{\max \{\lambda, \lambda\}}\left(p_{2 n}, p_{2 n+2}\right)+\delta_{\lambda}\left(p_{2 n+1}, p_{2 n+1}\right)}{2}\right\} \\
& \leq \max \left\{\delta_{\lambda}\left(p_{2 n}, p_{2 n+1}\right), \delta_{\lambda}\left(p_{2 n+1}, p_{2 n+2}\right),\right. \\
& \left.\frac{\delta_{\lambda}\left(p_{2 n}, p_{2 n+1}\right)+\delta_{\lambda}\left(p_{2 n+1}, p_{2 n+2}\right)}{2}\right\} \\
& =\max \left\{\delta_{\lambda}\left(p_{2 n}, p_{2 n+1}\right), \delta_{\lambda}\left(p_{2 n+1}, p_{2 n+2}\right)\right\} .
\end{aligned}
$$

If

$$
\max \left\{\delta_{\lambda}\left(p_{2 n}, p_{2 n+1}\right), \delta_{\lambda}\left(p_{2 n+1}, p_{2 n+2}\right)\right\}=\delta_{\lambda}\left(p_{2 n+1}, p_{2 n+2}\right)
$$

for each $n \in \mathbb{N}$, then from (3), we have a contradiction. So, $S\left(p_{2 n}, p_{2 n+1}\right)=\delta_{\lambda}\left(p_{2 n}, p_{2 n+1}\right)$ for all $n \in \mathbb{N}$. Consequently, (3) gives

$$
\delta_{\lambda}\left(p_{2 n+1}, p_{2 n+2}\right)<\delta_{\lambda}\left(p_{2 n}, p_{2 n+1}\right) .
$$

Hence for all $n \in \mathbb{N}$, continuing this way we get

$$
\delta_{\lambda}\left(p_{2 n}, p_{2 n+1}\right)<\delta_{\lambda}\left(p_{2 n-1}, p_{2 n-2}\right) .
$$

So $\left\{\delta_{\lambda}\left(p_{2 n}, p_{2 n+1}\right)\right\}$ is a monotonically decreasing sequence of nonnegative real numbers, and hence there exists $l \geq 0$ such that

$$
\lim _{n \rightarrow \infty} \delta_{\lambda}\left(p_{2 n}, p_{2 n+1}\right)=l .
$$

Assume that $l>0$. Then using (1) and (b) of the Definition 1.9, we have

$$
\begin{aligned}
C_{G} & \leq \limsup _{n \rightarrow \infty} \zeta\left(\delta_{\lambda}\left(K p_{2 n}, L p_{2 n+1}\right), S\left(p_{2 n}, p_{2 n+1}\right)\right) \\
& =\limsup _{n \rightarrow \infty} \zeta\left(\delta_{\lambda}\left(p_{2 n+1}, p_{2 n+2}\right), \delta_{\lambda}\left(p_{2 n}, p_{2 n+1}\right)\right)<C_{G},
\end{aligned}
$$


which is a contradiction and so $l=0$.

Now, we show that $\left\{p_{n}\right\}$ is a $\delta$-Cauchy sequence. It is sufficient to show that $\left\{p_{2 n}\right\}$ is a $\delta$-Cauchy sequence. Assume on the contrary. There exists $\varepsilon>0$ such that we can find two subsequences $\left\{m_{k}\right\}$ and $\left\{n_{k}\right\}$ of positive integers satisfying $n_{k}>m_{k} \geq k$ such the following inequalities hold:

$$
\delta_{\lambda}\left(p_{2 n_{k}}, p_{2 m_{k}}\right) \geq \varepsilon \text {, and } \delta_{\lambda}\left(p_{2 n_{k}-1}, p_{2 m_{k}}\right)<\varepsilon .
$$

From $(6)$ and $\left(\delta_{7}\right)$, it follows that

$$
\begin{aligned}
\varepsilon & \leq \delta_{\lambda}\left(p_{2 n_{k}}, p_{2 m_{k}}\right)=\delta_{\max \{\lambda, \lambda\}}\left(p_{2 n_{k}}, p_{2 m_{k}}\right) \\
& \leq \delta_{\lambda}\left(p_{2 n_{k}}, p_{2 n_{k}-1}\right)+\delta_{\lambda}\left(p_{2 n_{k}-1}, p_{2 m_{k}}\right) \\
& <\varepsilon+\delta_{\lambda}\left(p_{2 n_{k}}, p_{2 n_{k}-1}\right) .
\end{aligned}
$$

On taking limit as $k \rightarrow \infty$ in above relation, we obtain that

$$
\lim _{k \rightarrow \infty} \delta_{\lambda}\left(p_{2 n_{k}}, p_{2 m_{k}}\right)=\varepsilon
$$

Also, we have $\alpha\left(p_{2 n_{k}}\right) \beta\left(p_{2 m_{k}-1}\right) \geq 1$ and by (1),

$$
\begin{aligned}
C_{G} & \leq \zeta\left(\delta_{\lambda}\left(K p_{2 n_{k}}, L p_{2 m_{k}-1}\right), S\left(p_{2 n_{k}}, p_{2 m_{k}-1}\right)\right) \\
& =\zeta\left(\delta_{\lambda}\left(p_{2 n_{k}+1}, p_{2 m_{k}}\right), S\left(p_{2 n_{k}}, p_{2 m_{k}-1}\right)\right),
\end{aligned}
$$

where

$$
\begin{aligned}
S\left(p_{2 n_{k}}, p_{2 m_{k}-1}\right)= & \max \left\{\delta_{\lambda}\left(p_{2 n_{k}}, p_{2 m_{k}-1}\right), \delta_{\lambda}\left(p_{2 n_{k}}, K p_{2 n_{k}}\right),\right. \\
& \left.\delta_{\lambda}\left(p_{2 m_{k}-1}, L p_{2 m_{k}-1}\right), \frac{\delta_{\lambda}\left(p_{2 n_{k}}, L p_{2 m_{k}-1}\right)+\delta_{\lambda}\left(p_{2 m_{k}-1}, K p_{2 n_{k}}\right)}{2}\right\} \\
& =\max \left\{\delta_{\lambda}\left(p_{2 n_{k}}, p_{2 m_{k}-1}\right), \delta_{\lambda}\left(p_{2 n_{k}}, p_{2 n_{k}+1}\right),\right. \\
& \left.\delta_{\lambda}\left(p_{2 m_{k}-1}, p_{2 m_{k}}\right), \frac{\delta_{\lambda}\left(p_{2 n_{k}}, p_{2 m_{k}}\right)+\delta_{\lambda}\left(p_{2 m_{k}-1}, p_{2 n_{k}+1}\right)}{2}\right\} .
\end{aligned}
$$

Also, from (6) and $\left(\delta_{7}\right)$, it follows that

$$
\begin{aligned}
\delta_{\lambda}\left(p_{2 n_{k}+1}, p_{2 m_{k}}\right) & =\delta_{\max \{\lambda, \lambda\}}\left(p_{2 n_{k}+1}, p_{2 m_{k}}\right) \\
& \leq \delta_{\lambda}\left(p_{2 n_{k}+1}, p_{2 n_{k}}\right)+\delta_{\lambda}\left(p_{2 n_{k}}, p_{2 m_{k}}\right) \\
& =\delta_{\lambda}\left(p_{2 n_{k}+1}, p_{2 n_{k}}\right)+\delta_{\max \{\lambda, \lambda\}}\left(p_{2 n_{k}}, p_{2 m_{k}}\right) \\
& \leq \delta_{\lambda}\left(p_{2 n_{k}+1}, p_{2 n_{k}}\right)+\delta_{\lambda}\left(p_{2 n_{k}}, p_{2 n_{k}-1}\right) \\
& +\delta_{\lambda}\left(p_{2 n_{k}-1}, p_{2 m_{k}}\right) \\
& <\delta_{\lambda}\left(p_{2 n_{k}+1}, p_{2 n_{k}}\right)+\delta_{\lambda}\left(p_{2 n_{k}}, p_{2 n_{k}-1}\right)+\varepsilon, \\
\delta_{\lambda}\left(p_{2 m_{k}-1}, p_{2 n_{k}+1}\right) & =\delta_{\max \{\lambda, \lambda\}}\left(p_{2 m_{k}-1}, p_{2 n_{k}+1}\right) \\
& \leq \delta_{\lambda}\left(p_{2 m_{k}-1}, p_{2 m_{k}}\right)+\delta_{\lambda}\left(p_{2 m_{k}}, p_{2 n_{k}+1}\right) \\
& =\delta_{\lambda}\left(p_{2 m_{k}-1}, p_{2 m_{k}}\right)+\delta_{\max \{\lambda, \lambda\}}\left(p_{2 m_{k}}, p_{2 n_{k}+1}\right) \\
& \leq \delta_{\lambda}\left(p_{2 m_{k}-1}, p_{2 m_{k}}\right)+\delta_{\lambda}\left(p_{2 m_{k}}, p_{2 n_{k}-1}\right) \\
& \leq+\delta_{\lambda}\left(p_{2 n_{k}-1}, p_{2 n_{k}+1}\right) \\
& =\delta_{\lambda}\left(p_{2 m_{k}-1}, p_{2 m_{k}}\right)+\delta_{\lambda}\left(p_{2 m_{k}}, p_{2 n_{k}-1}\right) \\
& +\delta_{\max \{\lambda, \lambda\}}\left(p_{2 n_{k}-1}, p_{2 n_{k}+1}\right) \\
& \leq \delta_{\lambda}\left(p_{2 m_{k}-1}, p_{2 m_{k}}\right)+\delta_{\lambda}\left(p_{2 n_{k}-1}, p_{2 m_{k}}\right) \\
& +\delta_{\lambda}\left(p_{2 n_{k}-1}, p_{2 n_{k}}\right)+\delta_{\lambda}\left(p_{2 n_{k}}, p_{2 n_{k}+1}\right) \\
& <\varepsilon+\delta_{\lambda}\left(p_{2 m_{k}-1}, p_{2 m_{k}}\right)+\delta_{\lambda}\left(p_{2 n_{k}-1}, p_{2 n_{k}}\right) \\
& +\delta_{\lambda}\left(p_{2 n_{k}}, p_{2 n_{k}+1}\right),
\end{aligned}
$$


and

$$
\begin{aligned}
\delta_{\lambda}\left(p_{2 n_{k}}, p_{2 m_{k}-1}\right)= & \delta_{\max \{\lambda, \lambda\}}\left(p_{2 n_{k}}, p_{2 m_{k}-1}\right) \\
& \leq \delta_{\lambda}\left(p_{2 n_{k}}, p_{2 n_{k}-1}\right)+\delta_{\lambda}\left(p_{2 n_{k}-1}, p_{2 m_{k}-1}\right) \\
& =\delta_{\lambda}\left(p_{2 n_{k}}, p_{2 n_{k}-1}\right)+\delta_{\max \{\lambda, \lambda\}}\left(p_{2 n_{k}-1}, p_{2 m_{k}-1}\right) \\
& \leq \delta_{\lambda}\left(p_{2 n_{k}}, p_{2 n_{k}-1}\right)+\delta_{\lambda}\left(p_{2 n_{k}-1}, p_{2 m_{k}}\right) \\
& +\delta_{\lambda}\left(p_{2 m_{k}}, p_{2 m_{k}-1}\right) \\
& <\varepsilon+\delta_{\lambda}\left(p_{2 n_{k}}, p_{2 n_{k}-1}\right)+\delta_{\lambda}\left(p_{2 m_{k}}, p_{2 m_{k}-1}\right) .
\end{aligned}
$$

Moreover, because $(K, L)$ is a cyclic $(\alpha, \beta)$-admissible pair we attain $\alpha\left(p_{2 n_{k}}\right) \beta\left(p_{2 m_{k}-1}\right) \geq 1$. Then by (1),

$$
\begin{aligned}
C_{G} & \leq \zeta\left(\delta_{\lambda}\left(K p_{2 n_{k}}, L p_{2 m_{k}-1}\right), S\left(p_{2 n_{k}}, p_{2 m_{k}-1}\right)\right) \\
& =\zeta\left(\delta_{\lambda}\left(p_{2 n_{k}+1}, p_{2 m_{k}}\right), S\left(p_{2 n_{k}}, p_{2 m_{k}-1}\right)\right) \\
& <G\left(S\left(p_{2 n_{k}}, p_{2 m_{k}-1}\right), \delta_{\lambda}\left(p_{2 n_{k}+1}, p_{2 m_{k}}\right)\right) .
\end{aligned}
$$

Thence by (i) of Definition 1.10, we gain

$$
\delta_{\lambda}\left(p_{2 n_{k}+1}, p_{2 m_{k}}\right)<S\left(p_{2 n_{k}}, p_{2 m_{k}-1}\right) .
$$

Taking the limit as $k \rightarrow \infty$ and by using (6),(8), (10), (11), (12), (13), we procure

$$
\lim _{k \rightarrow \infty} \delta_{\lambda}\left(p_{2 n_{k}+1}, p_{2 m_{k}}\right)=\lim _{k \rightarrow \infty} S\left(p_{2 n_{k}}, p_{2 m_{k}-1}\right)=\varepsilon .
$$

Taking $s_{n}=\delta_{\lambda}\left(p_{2 n_{k}+1}, p_{2 m_{k}}\right), t_{n}=\lim _{k \rightarrow \infty} S\left(p_{2 n_{k}}, p_{2 m_{k}-1}\right)$ and using (13), (15) and (b) of Definition 1.9, we obtain

$$
\begin{aligned}
C_{G} & \leq \limsup _{k \rightarrow \infty} \zeta\left(\delta_{\lambda}\left(K p_{2 n_{k}}, L p_{2 m_{k}-1}\right), S\left(p_{2 n_{k}}, p_{2 m_{k}-1}\right)\right) \\
& =\limsup _{k \rightarrow \infty} \zeta\left(\delta_{\lambda}\left(p_{2 n_{k}+1}, p_{2 m_{k}}\right), S\left(p_{2 n_{k}}, p_{2 m_{k}-1}\right)\right)<C_{G} .
\end{aligned}
$$

The attained last inequality causes a contradiction. Accordingly $\left\{p_{2 n}\right\}$ is a $\delta$-Cauchy sequence.

As $M_{\delta}$ is a $\delta$-complete non-Archimedean modular metric space, there exists $u \in M_{\delta}$ such that $\delta_{\lambda}\left(p_{n}, u\right) \rightarrow 0$ as $n \rightarrow \infty$.

Now we present $u$ is a common fixed point of $K$ and $L$. From (iii), we will presume that $K$ is a $\delta$-continuous mapping. Since $\delta_{\lambda}\left(p_{2 n}, j\right) \rightarrow 0$ as $n \rightarrow \infty$ and $K$ is a $\delta$-continuous mapping, we get

$$
\delta_{\lambda}\left(p_{2 n+1}, j\right)=\delta_{\lambda}\left(K p_{2 n+1}, K j\right) \rightarrow 0,
$$

as $n \rightarrow \infty$. Hence $p_{2 n+1} \rightarrow K j$ as $n \rightarrow \infty$. But $p_{2 n+1} \rightarrow j$ as $n \rightarrow \infty$. By the uniqueness of the limit, we achieve $K j=j$.

From (iv), we have $\beta(j) \geq 1$. This implies that $\alpha\left(p_{2 n}\right) \beta(j) \geq 1$ for all $n \in \mathbb{N}$. Next, we will demonstrate that $j$ is a fixed point of $L$. We presume that $j \neq K j$, that is $\delta_{\lambda}(j, K j)>0$. By $K$ and $L$ are generalized $(\alpha, \beta)$-simulation contraction, we attain

$$
C_{G} \leq \zeta\left(\delta_{\lambda}\left(K p_{2 n}, L j\right), S\left(p_{2 n}, j\right)\right)<G\left(S\left(p_{2 n}, j\right), \delta_{\lambda}\left(K p_{2 n}, L j\right)\right)
$$

and then by (i) of Definition 1.10, we procure

$$
\delta_{\lambda}\left(K p_{2 n}, L j\right)<S\left(p_{2 n}, j\right) .
$$

Taking limit as $n \rightarrow \infty$ in (18), we have

$$
\delta_{\lambda}(j, L j)<\delta_{\lambda}(j, L j),
$$

which is a contradiction. Thus $j=L j$ and hence $j$ is a common fixed point $K$ and $L$.

Ultimately, we will demonstrate the uniqueness of a common fixed point of $K$ and $L$. We assume that $s$ is another common fixed point, that is, $\delta_{\lambda}(j, s) \neq 0$. From hypothesis, we gain $\alpha(j) \geq 1$ and $\beta(s) \geq 1$ and thus $\alpha(j) \beta(s) \geq 1$. By using (1),

$$
C_{G} \leq \zeta\left(\delta_{\lambda}(K j, L s), S(j, s)\right)<G\left(S(j, s), \delta_{\lambda}(K j, L s)\right)
$$


and from (i) of Definition 1.10, we have

$$
\begin{aligned}
& \delta_{\lambda}(K j, L s)<S(j, s)
\end{aligned}
$$

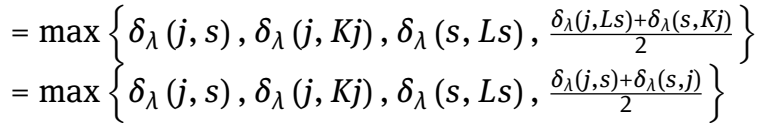

$$
\begin{aligned}
& =\max \left\{\delta_{\lambda}(j, s), 0\right\} \\
& =\delta_{\lambda}(j, s) \text {, }
\end{aligned}
$$

which is a contradiction. Hence $j=s$.

\section{Consequences}

This section aims to acquaint some consequences from the main theorem.

Corollary 3.1 Let $M_{\delta}$ be a $\delta$-complete non-Archimedean modular metric space and $K, L: M_{\kappa} \rightarrow M_{\delta}$ be two mappings. Presume that there exists $k \in(0,1)$ such that

$$
\begin{aligned}
& p, q \in M_{\kappa} \text { with } \quad \alpha(p) \beta(q) \geq 1 \Rightarrow \\
& \delta_{\lambda}(K p, L q) \leq k \max \left\{\delta_{\lambda}(p, q), \delta_{\lambda}(p, K p), \delta_{\lambda}(q, L q), \frac{\delta_{\lambda}(p, L q)+\delta_{\lambda}(q, K p)}{2}\right\} .
\end{aligned}
$$

Suppose that the following circumstances hold:

i. $(K, L)$ is a cyclic $(\alpha, \beta)$-admissible pair,

ii. there exists $p_{0} \in M_{\delta}$ such that $\alpha\left(p_{0}\right) \geq 1$,

iii. $K$ or $L$ is a $\delta$-continuous mapping,

iv. if $\left\{p_{n}\right\}$ is a sequence in $M_{\delta}$ such that $p_{n} \rightarrow \xi$ and $\alpha\left(p_{2 n}\right) \geq 1$ and $\beta\left(p_{2 n-1}\right) \geq 1$ for all $n \in \mathbb{N}$, then $\alpha(p) \geq 1$ and $\beta(p) \geq 1$.

Then, $K$ and $L$ admit a common fixed point.

Corollary 3.2 Let $M_{\delta}$ be a complete non-Archimedean modular metric space and $K, L: M_{\delta} \rightarrow M_{\delta}$ be two mappings. Assume that there exists a continuous function $\varphi:[0, \infty) \rightarrow[0, \infty)$ with $\varphi(t)=0$ if and only if $t=0$ such that

$$
\begin{aligned}
p, q \in M_{\delta} \text { with } \quad \alpha(p) \beta(q) \geq 1 \quad \Rightarrow \\
\delta_{\lambda}(K p, L q) \leq \max \left\{\delta_{\lambda}(p, q), \delta_{\lambda}(p, K p), \delta_{\lambda}(q, L q), \frac{\delta_{\lambda}(p, L q)+\delta_{\lambda}(q, K p)}{2}\right\} \\
-\varphi\left(\max \left\{\delta_{\lambda}(p, q), \delta_{\lambda}(p, K p), \delta_{\lambda}(q, L q), \frac{\delta_{\lambda}(p, L q)+\delta_{\lambda}(q, K p)}{2}\right\}\right) .
\end{aligned}
$$

Suppose that the following conditions hold:

i. $(K, L)$ is a cyclic $(\alpha, \beta)$-admissible pair,

ii. there exists $p_{0} \in M_{\delta}$ such that $\alpha\left(p_{0}\right) \geq 1$,

iii. $K$ or $L$ is a $\delta$-continuous mapping,

iv. if $\left\{p_{n}\right\}$ is a sequence in $M_{\delta}$ such that $p_{n} \rightarrow p$ and $\alpha\left(p_{2 n}\right) \geq 1$ and $\beta\left(p_{2 n-1}\right) \geq 1$ for all $n \in \mathbb{N}$, then $\alpha(p) \geq 1$ and $\beta(p) \geq 1$.

Then, $K$ and $L$ admit a common fixed point.

We will replace the condition of cyclic $(\alpha, \beta)$-admissible pair with cyclic $(\alpha, \beta)$-admissible mapping in our main result, that is, we will take $K=L$. Then, we have the following results.

Corollary 3.3 Let $M_{\delta}$ be a complete non-Archimedean modular metric space and $K: M_{\delta} \rightarrow M_{\delta}$ be a given mapping. Assume that there exists $\zeta \in T_{G}$ such that

$$
\begin{aligned}
& p, q \in M_{\delta} \text { with } \quad \alpha(p) \beta(q) \geq 1 \Rightarrow \\
& \zeta\left(\delta_{\lambda}(K p, L q), \max \left\{\delta_{\lambda}(p, q), \delta_{\lambda}(p, K p), \delta_{\lambda}(q, L q), \frac{\delta_{\lambda}(p, K q)+\delta_{\lambda}(q, K p)}{2}\right\}\right) \geq C_{G} .
\end{aligned}
$$

Suppose that the following conditions hold:

i. $\quad K$ is a cyclic $(\alpha, \beta)$-admissible mapping; 
ii. there exists $p_{0} \in M_{\delta}$ such that $\alpha\left(p_{0}\right) \geq 1$ and $\beta\left(p_{0}\right) \geq 1$;

iii. $V$ is a $\delta$-continuous mapping, or;

iv. if $\left\{p_{n}\right\}$ is a sequence in $M_{\delta}$ such that $p_{n} \rightarrow p$ and $\beta\left(p_{n}\right) \geq 1$ for all $n \in \mathbb{N}$, then $\beta(p) \geq 1$.

Then, $K$ admits a fixed point.

Corollary 3.4 Let $M_{\delta}$ be a complete non-Archimedean modular metric space and $K: M_{\delta} \rightarrow M_{\delta}$ be a given mapping. Assume that there exists $\zeta \in T_{G}$ such that

$$
\alpha(p) \beta(q) \geq 1 \quad \Rightarrow \quad \zeta\left(\delta_{\lambda}(K p, K q), \delta_{\lambda}(p, q)\right) \geq C_{G},
$$

$p, q \in M_{\delta}$. Suppose that the following conditions hold:

i. $\quad K$ is a cyclic $(\alpha, \beta)$-admissible mapping;

ii. there exists $p_{0} \in M_{\delta}$ such that $\alpha\left(p_{0}\right) \geq 1$ and $\beta\left(p_{0}\right) \geq 1$;

iii. $K$ is a $\delta$-continuous mapping, or;

iv. if $\left\{p_{n}\right\}$ is a sequence in $M_{\delta}$ such that $p_{n} \rightarrow \xi$ and $\beta\left(p_{n}\right) \geq 1$ for all $n \in \mathbb{N}$, then $\beta(p) \geq 1$.

Then, $K$ admits a fixed point.

Example 3.5 Let $M_{\delta}=\mathbb{R}, \delta_{\lambda}(p, q)=\frac{1}{\lambda}|p-q|$ for all $p \in M_{\delta}$. Define the mapping $K: M_{\delta} \rightarrow M_{\delta}$ as follows:

$$
K p= \begin{cases}\frac{p}{4}, & \text { if } p \in[0,1] \\ p^{2}, & \text { if } p>1 .\end{cases}
$$

and $\alpha, \beta: M_{\delta} \rightarrow[0,+\infty)$ are defined by

$$
\alpha(p)=\beta(p)= \begin{cases}1, & p \in[0,1] \\ 0, & \text { otherwise }\end{cases}
$$

If we consider the functions $\zeta(p, q)=\frac{1}{3} q-p, \quad G(q, p)=q-p$ and $C_{G}=\frac{p \cdot q}{12^{a}}, a>2$, then all the hypotheses of Corollary 3.4 are satisfied and $p=0$ is a unique fixed point of $K$.

On the other hand, $\delta_{\lambda}(K \sqrt{6}, K \sqrt{5})=\frac{1}{\lambda}>\frac{\sqrt{6}-\sqrt{5}}{\lambda}=\delta_{\lambda}(\sqrt{6}, \sqrt{5}), \lambda>0$. Thus $K$ is not a Banach contraction with respect to $M_{\delta}$.

\section{Common fixed points on non-Archimedean modular metric spaces with a directed graph}

Let $M_{\delta}$ be a non-Archimedean modular metric space and $\Delta=\{(p, p): p \in M\}$ denotes the diagonal of $M_{\delta} \times M_{\delta}$. Let $Z$ be a directed graph such that the set $V(Z)$ of its vertices coincides with $M$ and $J(Z)$ be the set of edges of the graph such that $\Delta \subseteq J(Z)$.

Let $M_{\delta}$ be a non-Archimedean modular metric space endowed with a graph $Z$ and $K, L: M_{\delta} \rightarrow M_{\delta}$. We set

$$
M_{K L}=\{p \in M:(p, K p) \in J(Z) \quad \text { and } \quad(K p, L K p) \in J(Z)\} .
$$

Definition 4.1 Let $M_{\delta}$ be a non-Archimedean modular metric space endowed with a directed graph $Z$ and $(K, L)$ be a cyclic $(\alpha, \beta)$ admissible pair. We say that $K$ and $L$ are generalized $(\alpha, \beta)$-Z-simulation contraction if there exists $\zeta \in T_{G}$ such that

$$
\alpha(p) \beta(\eta) \geq 1 \Rightarrow \zeta\left(\delta_{\lambda}(K p, L q), S(p, q)\right) \geq C_{G},
$$

where $S(p, q)=\max \left\{\delta_{\lambda}(p, q), \delta_{\lambda}(p, K p), \delta_{\lambda}(q, L q), \frac{\kappa_{\lambda}(p, L q)+\delta_{\lambda}(q, L p)}{2}\right\}$, for all $(p, q) \in J(Z)$.

Theorem 4.2 Let $M_{\delta}$ be a $\delta$-complete non-Archimedean modular metric space endowed with a directed graph $Z$. $K$ and $L$ are generalized $(\alpha, \beta)-Z$-simulation contraction. Suppose that the following conditions hold:

i. there exists $p_{0} \in M_{K L}$ such that $\alpha\left(p_{0}\right) \geq 1$;

ii. if $\left\{p_{n}\right\}$ is a sequence in $M$ such that $p_{n} \rightarrow p,\left(p_{2 n}, p_{2 n+1}\right) \in J(Z)$ for all $n \geq 0$; 
$i i_{a} . L$ is $\delta$-continuous, $\left(p, p_{2 n_{k}+1}\right) \in J(Z)$ for all $k \geq 0$ and $\alpha\left(p_{2 n}\right) \geq 1$ then $\alpha(p) \geq 1$, or;

$i i_{b}$. $K$ is $\delta$-continuous, $\left(p_{2 n_{k}}, p\right) \in J(Z)$ for all $k \geq 0$ and $\beta\left(p_{2 n+1}\right) \geq 1$, then $\beta(p) \geq 1$;

iii. there is a sequence $\left\{p_{n}\right\}_{n \in \mathbb{N}}$ in $M_{\delta}$, such that

$$
\left(p_{2 n}, K p_{2 n}\right) \in J(Z) \quad \Rightarrow \quad\left(p_{2 n+2}, K p_{2 n+2}\right) \in J(Z)
$$

and

$$
\left(p_{2 n+1}, L p_{2 n+1}\right) \in J(Z) \quad \Rightarrow \quad\left(p_{2 n+3}, L p_{2 n+3}\right) \in J(Z) ;
$$

Then $K$ and $L$ hold a common fixed point.

Proof Let $p_{0}$ be a given point in $M_{K L}$, then $\left(p_{0}, K p_{0}\right) \in J(Z)$ and $\left(K p_{0}, L K p_{0}\right) \in J(Z)$, that is, $\left(p_{0}, p_{1}\right) \in$ $J(Z)$ and $\left(p_{1}, L p_{1}\right)=\left(p_{1}, p_{2}\right) \in J(Z)$. From (iii), it follows that $\left(p_{2}, K p_{2}\right) \in J(Z)$ and $\left(p_{3}, L p_{3}\right) \in J(Z)$. That is, $\left(p_{2}, p_{3}\right) \in J(Z)$ and $\left(p_{3}, p_{4}\right) \in J(Z)$. Continuing this way, we can obtain a sequence $\left\{p_{n}\right\}$ in $M_{\delta}$ such that $\left(p_{2 n}, K p_{2 n}\right) \in J(Z)$ and $\left(p_{2 n+1}, L p_{2 n+1}\right) \in J(Z)$ for all $n \in \mathbb{N}$. Also, $\left(p_{2 n}, p_{2 n+1}\right) \in J(Z)$ and $\left(p_{2 n+1}, p_{2 n+2}\right) \in J(Z)$ for all $n \in \mathbb{N}$. Using arguments as in the proof of the Theorem 2.2, we obtain that

$$
\delta_{\lambda}\left(p_{n}, p_{n+1}\right) \rightarrow 0 \quad \text { as } \quad \mathrm{n} \rightarrow \infty
$$

and $\left\{p_{n}\right\}$ is $\delta$-Cauchy sequence. Since $M_{\delta}$ is a $\delta$-complete, there exists a $p^{\star} \in M_{\delta}$ such that

$$
\delta_{\lambda}\left(p_{n}, p^{\star}\right) \rightarrow 0 \quad \text { as } \quad \mathrm{n} \rightarrow \infty .
$$

Now we show that $p^{\star}$ is a common fixed point. As $\delta_{\lambda}\left(p_{n}, p^{\star}\right) \rightarrow 0$ and $\left(p_{2 n}, p_{2 n+1}\right) \in J(Z)$, so there exists a subsequence $\left\{p_{2 n_{k}}\right\}$ of $\left\{p_{2 n}\right\}$ such that from (ii) either $L$ is $\delta$-continuous, $\left(p^{\star}, p_{2 n_{k}+1}\right) \in J(Z)$ and $\alpha\left(p^{*}\right) \geq 1$ or $K$ is $\delta$-continuous, $\left(p_{2 n_{k}}, p^{*}\right) \in J(Z)$ and $\beta\left(p^{*}\right) \geq 1$. Assume that $L$ is $\delta$-continuous, $\left(p^{\star}, p_{2 n_{k}+1}\right) \in J(Z)$ and $\alpha\left(p^{\star}\right) \geq 1$. Thus

$$
\delta_{\lambda}\left(L p_{2 n_{k}+1}, L p^{\star}\right)=\delta_{\lambda}\left(p_{2 n_{k}+2}, L p^{\star}\right) \rightarrow 0 \quad \text { as } \quad k \rightarrow \infty .
$$

This implies that $L p^{\star}=p^{\star}$. From Definition 4.1, we have $\alpha\left(p^{\star}\right) \beta\left(p_{2 n_{k}+1}\right) \geq 1$

$$
\begin{aligned}
C_{G} & \leq \zeta\left(\delta_{\lambda}\left(K p^{\star}, L p_{2 n_{k}+1}\right), S\left(p^{\star}, p_{2 n_{k}+1}\right)\right) \\
& <G\left(S\left(p^{\star}, p_{2 n_{k}+1}\right), \delta_{\lambda}\left(K p^{\star}, L p_{2 n_{k}+1}\right)\right)
\end{aligned}
$$

and then by (i) of Definition 1.10, we get

$$
\delta_{\lambda}\left(K p^{\star}, L p_{2 n_{k}+1}\right)<S\left(p^{\star}, p_{2 n_{k}+1}\right) .
$$

where

$$
\begin{aligned}
S\left(p^{\star}, p_{2 n_{k}+1}\right)= & \max \left\{\delta_{\lambda}\left(p^{\star}, p_{2 n_{k}+1}\right), \delta_{\lambda}\left(p^{\star}, K p^{\star}\right), \delta_{\lambda}\left(p_{2 n_{k}+1}, L p_{2 n_{k}+1}\right),\right. \\
& \left.\delta_{\lambda}\left(p^{\star}, L p_{2 n_{k}+1}\right), \delta_{\lambda}\left(p_{2 n_{k}+1}, K p^{\star}\right)\right\} .
\end{aligned}
$$

On taking the limit as $k \rightarrow \infty$ on both sides of inequality (23), we have

$$
\delta_{\lambda}\left(p^{\star}, K p^{\star}\right)<\delta_{\lambda}\left(p^{\star}, K p^{\star}\right),
$$

a contradiction. Thus $K p^{*}=p^{\star}$, and hence $p^{*}$ is a common fixed point. Similarly, the result follows if we suppose that $K$ is $\delta$-continuous, $\left(p_{2 n_{k}}, p^{\star}\right) \in J(Z)$ and $\beta\left(p^{\star}\right) \geq 1$.

We note that Theorem 4.2 does not guarantee the uniqueness of a common fixed point.

To obtain the uniqueness, additional assumptions as given in the following theorem are needed.

Theorem 4.3 Let $M_{\delta}$ be a $\delta$-complete non-Archimedean modular metric space endowed with a directed graph $Z$. $K$ and $L$ are generalized $(\alpha, \beta)$ - $Z$-simulation contraction. Suppose that the following conditions hold:

$i$. there exists $p_{0} \in M_{K L}$ such that $\alpha\left(p_{0}\right) \geq 1$;

ii. if $\left\{p_{n}\right\}$ is a sequence in $M_{\delta}$ such that $p_{n} \rightarrow p,\left(p_{2 n}, p_{2 n+1}\right) \in J(Z)$ for all $n \geq 0$;

$i i_{a}$. $L$ is $\delta$-continuous, $\left(p, p_{2 n_{k}+1}\right) \in J(Z)$ for all $k \geq 0$ and $\alpha\left(p_{2 n}\right) \geq 1$ then $\alpha(p) \geq 1$, or;

$i i_{b}$. $K$ is $\delta$-continuous, $\left(p_{2 n_{k}}, p\right) \in J(Z)$ for all $k \geq 0$ and $\beta\left(p_{2 n+1}\right) \geq 1$, then $\beta(p) \geq 1$; 
iii. there is a sequence $\left\{p_{n}\right\}_{n \in \mathbb{N}}$ in $M_{\delta}$, such that

$$
\left(p_{2 n}, K p_{2 n}\right) \in J(Z) \quad \Rightarrow \quad\left(p_{2 n+2}, K p_{2 n+2}\right) \in J(Z)
$$

and

$$
\left(p_{2 n+1}, L p_{2 n+1}\right) \in J(Z) \quad \Rightarrow \quad\left(p_{2 n+3}, L p_{2 n+3}\right) \in J(Z) ;
$$

iv. there exist $p^{\star}, q^{\star} \in \operatorname{Fix}(K, L)$ such that $\left(p^{\star}, q^{\star}\right) \in J(Z), \alpha\left(p^{\star}\right) \geq 1$ and $\beta\left(q^{\star}\right) \geq 1$.

Then $K$ and $L$ hold a unique common fixed point.

Proof Let $p^{\star}, q^{\star}$ be common fixed points of $K$ and $L$, then $\left(p^{\star}, q^{\star}\right) \in J(Z)$. Also, since $\alpha\left(p^{\star}\right) \geq 1$ and $\beta\left(q^{\star}\right) \geq 1$. Thus, by Definition 4.1, we get that

$$
C_{G} \leq \zeta\left(\delta_{\lambda}\left(K p^{\star}, L q^{\star}\right), S\left(p^{\star}, q^{\star}\right)\right)<G\left(S\left(p^{\star}, q^{\star}\right), \delta_{\lambda}\left(K p^{\star}, L q^{\star}\right)\right)
$$

and from (i) of the Definition 1.10, we have

$$
\begin{aligned}
\delta_{\lambda}\left(K p^{\star}, L q^{\star}\right) & <S\left(p^{\star}, q^{\star}\right) \\
& =\max \left\{\delta_{\lambda}\left(p^{\star}, q^{\star}\right), 0\right\} \\
& =\delta_{\lambda}\left(p^{\star}, q^{\star}\right) .
\end{aligned}
$$

which is a contradiction. Hence $p^{\star}=q^{\star}$.

Example 4.4 Let $M_{\delta}=[0,1], \delta_{\lambda}(p, q)=\frac{1}{\lambda}|p-q|$ for all $p \in M_{\delta}$ and $E(G)=\{(p, q): p, q \in[0,1]\}$. Define the mappings $K, L: M \rightarrow M$ as follows:

$$
K p=\frac{p}{2}, \quad L p=\frac{p}{3}, \quad p \in M_{\delta} .
$$

and $\alpha, \beta: M_{\delta} \rightarrow[0,+\infty)$ are defined by

$$
\alpha(p)=\beta(p)= \begin{cases}1, & p \in(0,1) \\ 0, & \text { otherwise }\end{cases}
$$

If we consider the functions $\zeta(p, q)=\frac{3}{4} q-p, \quad G(q, p)=q-p$ and $C_{G}=\frac{p \cdot q}{24^{a}}, \quad a>2$, then all the hypotheses of Theorem 4.3 are satisfied and $p=0$ is a unique common fixed point.

\section{Application to dynamic programming}

The purpose of this section is to present an application on dynamic programming.

Throughout this section, we assume that $A$ and $B$ are Banach spaces, $W \subseteq A$ and $D \subseteq B$ is a decision space. Now, consider the following system of functional equations:

$$
\begin{aligned}
& P(p)=\sup _{q \in D}\{s(p, q)+\chi(p, q, P(\tau(p, q)))\}, \\
& Q(p)=\sup _{q \in D}\{s(p, q)+\varsigma(p, q, Q(\tau(p, q)))\},
\end{aligned}
$$

where $\tau: W \times D \rightarrow W, r: W \times D \rightarrow \mathbb{R}, \chi: W \times D \times \mathbb{R} \rightarrow \mathbb{R}, \varsigma: W \times D \times \mathbb{R} \rightarrow \mathbb{R}$.

Let $M=B(W)$ denotes the space of all bounded real-valued function on $W$. Consider the metric given by

$$
\delta_{\lambda}(m, n)=\frac{1}{\lambda} \sup _{p \in W}|m(p)-n(p)| \text {, for all } m, n \in A \text { and } \lambda>0 .
$$

Then $M_{\delta}$ is a $\delta$-complete non-Archimedean modular metric space.

Now, take the mappings $E, F: M_{\delta} \rightarrow M_{\delta}$ as

$$
E m(p)=\sup _{q \in D}\{s(p, q)+\chi(p, q, m(\tau(p, q)))\},
$$


and

$$
F m(p)=\sup _{q \in D}\{s(p, q)+\varsigma(p, q, m(\tau(p, q)))\},
$$

where $p \in W$ and $m \in M_{\delta}$. If the functions $s, \chi, \tau$ are bounded, then $E$ and $F$ are well-defined.

Theorem 5.1 Suppose that there exists $k \in[0,1)$ such that for each $(p, q) \in W \times D$ and $m_{1}, m_{2} \in M_{\delta}$, the inequality

$$
\left|\chi\left(p, q, m_{1}(\tau(p, q))\right)-\varsigma\left(p, q, m_{2}(\tau(p, q))\right)\right| \leq k\left|m_{1}-m_{2}\right|,
$$

holds. Then, $E$ and $F$ admit a common fixed point in $M_{\delta}$.

Proof Let $\varepsilon>0$ be an arbitrary real number, $p \in W$ and $m_{1}, m_{2} \in M_{\delta}$. Then by (28) and (29), there exist $q_{1}, q_{2} \in D$ such that

$$
\begin{gathered}
E m_{1}(p)<s\left(p, q_{1}\right)+\chi\left(p, q_{1}, m_{1}\left(\tau\left(p, q_{1}\right)\right)\right)+\varepsilon, \\
F m_{2}(p)<s\left(p, q_{2}\right)+s\left(p, q_{2}, m_{2}\left(\tau\left(p, q_{2}\right)\right)\right)+\varepsilon, \\
\operatorname{Em}_{1}(p) \geq s\left(p, q_{2}\right)+\chi\left(p, q_{2}, m_{1}\left(\tau\left(p, q_{2}\right)\right)\right),
\end{gathered}
$$

and

$$
F m_{2}(p) \geq s\left(p, q_{1}\right)+\varsigma\left(p, q_{1}, m_{2}\left(\tau\left(p, q_{1}\right)\right)\right) .
$$

Then from (30) and (33), it follows easily that

$$
\begin{aligned}
E m_{1}(p)-F m_{2}(p) & \leq \chi\left(p, q_{1}, m_{1}\left(\tau\left(p, q_{1}\right)\right)\right)+\varepsilon-\varsigma\left(p, q_{1}, m_{2}\left(\tau\left(p, q_{1}\right)\right)\right) \\
& \leq\left|\chi\left(p, q_{1}, m_{1}\left(\tau\left(p, q_{1}\right)\right)\right)-\varsigma\left(p, q_{1}, m_{2}\left(\tau\left(p, q_{1}\right)\right)\right)\right|+\varepsilon \\
& \leq k\left|m_{1}-m_{2}\right|+\varepsilon .
\end{aligned}
$$

Similarly, from (31) and (32), we have

$$
F m_{2}(p)-E m_{1}(p) \leq k\left|m_{1}-m_{2}\right|+\varepsilon .
$$

Thus, we deduce from above inequalities that

$$
\left|E m_{1}(p)-F m_{2}(p)\right| \leq k\left|m_{1}-m_{2}\right|+\varepsilon .
$$

Since the inequality (34) is valid for any $p \in W$, then

$$
\delta_{\lambda}\left(E m_{1}, F m_{2}\right)=\frac{1}{\delta}\left|E m_{1}-F m_{2}\right| \leq \frac{1}{\delta} k\left|m_{1}-m_{2}\right|+\varepsilon=k \delta_{\lambda}\left(m_{1}, m_{2}\right)+\varepsilon,
$$

also $\varepsilon>0$ is arbitrary, so

$$
\delta_{\lambda}\left(E m_{1}, F m_{2}\right) \leq k \delta_{\lambda}\left(m_{1}, m_{2}\right) .
$$

Now, taking $\zeta(a, b)=k b-a, \quad G(b, a)=b-a$, then for $C_{G}=0, \quad S(p, q)=\delta_{\lambda}(p, q)$, and $\alpha(p) \beta(q)=1$ in Theorem 2.2. Hence all conditions of Theorem 2.2 are satisfied. Therefore $E$ and $F$ have a common fixed point, that is, the functional equation (26) and (27) have a common solution in $M_{\delta}$.

Acknowledgments: The authors would like to thank the referees for the valuable comments and suggestions on this manuscript.

\section{References}

[1] V. V. Chistyakov, Modular metric spaces, I: Basic concepts, Nonlinear Anal., 72 (2010), 1-14.

[2] V. V. Chistyakov, Modular metric spaces, II: Application to superposition operators, Nonlinear Anal., 72 (2010), 15-30.

[3] M. Paknazar, M.A. Kutbi, M. Demma, P. Salimi, On non-Archimedean modular metric space and some nonlinear contraction mappings, Available online: https://pdfs.semanticscholar.org/ (accessed on 30 October 2019). 
[4] C. Mongkolkeha, W. Sintunavarat, P. Kumam, Fixed point theorems for contraction mappings in modular metric spaces, Fixed Point Theory Appl., 2011: 93 (2011), https://doi.org/10.1186/1687-1812-2011-93.

[5] F. Khojasteh, S. Shukla, S. Radenovic, A new approach to the study of fixed point theorems via simulation functions, Filomat, 29 (2015), no. 6, 1189-1194.

[6] H. Argoubi, B. Samet, C. Vetro, Nonlinear contractions involving simulation functions in a metric space with a partial order, J. Nonlinear Sci. Appl., 8 (2015), 1082-1094.

[7] M. Abbas, A. Latif, Y. Suleiman Fixed points for cyclic $R$-contractions and solution of nonlinear Volterra integro-differantial equations, Fixed Point Theory Appl., 2016:61 (2016), https://doi.org/10.1186/s13663-016-0552-1.

[8] M. Asadi, M. Gabeleh, C. Vetro, A new approach to the generalization of Darbo's fixed point problem by using simulation functions with application to integral equations, Result Math., 78:86 (2019), https://doi.org/10.1007/s00025-019-1010-2.

[9] M. Asadi, M. Azhini, E. Karapınar, H. Monfared, Simulation functions over $M$-metric spaces, East Asian Math. Journal, 33(2015), no. 5, 559-570.

[10] M. Monfared, M. Asadi, M. Gabeleh, A. Farajzadeh, New generalization of Darbo's fixed point theorem via $\alpha$-admissible simulation functions with application, Sahand Communications in Mathematical Analysis, 17(2020), no. 2, 161-171.

[11] B. Samet, Best proximity point results in partially ordered metric spaces via simulation functions, Fixed Point Theory Appl., 2015:232 (2015), https://doi.org/10.1186/s13663-015-0484-1.

[12] F. Tchier, C. Vetro, F. Vetro, Best approximation and variational inequality problems involving a simulation function, Fixed Point Theory Appl., 2016:26 (2016), https://doi.org/10.1186/s13663-016-0512-9.

[13] A.H. Ansari, Note on $\varphi-\psi$-contractive type mappings and related fixed point, in roceedings of the Second Regional Conference on Mathematical Sciences and Applications, (Tonekabon, Iran), 2014 September, Payame Noor University, (2014), 377-380.

[14] S. Radenovic, S. Chandok, Simulation type functions and coincidence point results, Filomat, 32 (2018), no. 1, 141-147.

[15] S. Alizadeh, F. Moradlou, P. Salimi, Some fixed point results for $(\alpha, \beta)-(\psi, \phi)$-contractive mappings, Filomat, 28 (2014), no. 3, 635-647.

[16] A.Latif, A.H. Ansari, Fixed points and functional equation problems via cyclic admissible generalized contractive type mappings , J. Nonlinear Sci. Appl., 9 (2016), 1129-1142. 